\title{
Corticotropin-releasing hormone and copeptin as acute stress markers in serial cerebrospinal fluid - first evidence for non- response during insulin hypoglycemia test
}

\section{Authors:}

\section{Corresponding author}

Viola Wortmann, M.D.

Department of Psychiatry and Psychotherapy, University Hospital Hamburg-Eppendorf, Martinistrasse 52, W37, D-20246 Hamburg, Germany.

\section{Coauthors:}

David Kober, M.D., University Hospital Hamburg-Eppendorf, Department of Anesthesiology, Hamburg, Germany; E-mail: d.kober@uke.de

Mersija Pulic, University Hospital Hamburg-Eppendorf, Department of Psychiatry and Psychotherapy, Hamburg, Germany; E-mail: mersija7@googlemail.com

Kai Boelmans, M.D., Ph.D., University Hospital Hamburg-Eppendorf, Department of Psychiatry and Psychotherapy, Hamburg, Germany; E-mail: boelmans_k@ukw.de

Martin Petzoldt, M.D., University Hospital Hamburg-Eppendorf, Department of Anesthesiology, Hamburg, Germany; E-mail: m.petzoldt@uke.de

Günter K. Stalla, M.D., Ph.D., Max Planck Institute of Psychiatry, Munich, Germany; E-mail: $\underline{\text { stalla@psych.mpg.de }}$

Klaus Wiedemann, M.D., Ph.D., University Hospital Hamburg-Eppendorf, Department of Psychiatry and Psychotherapy, Hamburg, Germany; E-mail: wiedemann@uke.de

Michael Kellner, M.D., Ph.D., University Hospital Hamburg-Eppendorf, Department of Psychiatry and Psychotherapy, Hamburg, Germany; E-mail: kellner@uke.de

There are no conflicts of interests to declare. 


\begin{abstract}
$\underline{\text { Abstract }}$
Background: During stress, arginine vasopressin (AVP) and corticotropin-releasing hormone $(\mathrm{CRH})$ can act as potent hypothalamic stimulators of the hypothalamic pituitary adrenocortical (HPA) axis. Recently, plasma CT-proAVP, also termed copeptin, was found to be a stable and sensitive surrogate marker for AVP release. A valid assessment of both CRH and copeptin in cerebrospinal fluid (CSF) might directly quantify an individual's current stress level. Here we investigated how concentrations of $\mathrm{CRH}$ and copeptin in CSF alter during insulininduced hypoglycemia test (IHT) - which has been shown to activate both hypothalamic AVP and $\mathrm{CRH}$ - and hypothesized an increase of both.

Methods: Five healthy young men were studied from 08:00 until 14:00 after over-night fasting. They received an i.v. injection of human insulin $(0.1 \mathrm{IE} / \mathrm{kg}$ body weight) at 11:02. CSF samples were drawn from a subarachnoidal catheter every 20 minutes from 10:40 to 14:00 for the measurement of CSF CRH and CSF copeptin. Plasma adrenocorticotropic hormone (ACTH) and cortisol were analyzed in parallel.

Results: Data could be assessed in three of five subjects, of which two responded to IHT by showing glucose levels of $<40 \mathrm{mg} / \mathrm{dl}$ and clinical symptoms (sweating). Despite a 17-fold, respectively 11-fold increase of plasma ACTH, neither our hypothesized increase of CSF CRH nor of CSF copeptin was seen in these two responders.

Conclusions: This is the first study investigating CSF CRH and CSF copeptin in man during acute stress. Copeptin had not been measured in CSF before. Our results support recent speculations that CSF CRH is not a measure of hypothalamic but rather extrahypothalamic CRH (non-HPA CRH). Further research on copeptin in CSF is needed.
\end{abstract}

Keywords: corticotropin releasing hormone (CRH), copeptin, serial cerebrospinal fluid, hypothalamic-pituitary-adrenal axis, stress, insulin-induced hypoglycemia test. 
Medical Research Archives, Vol. 5, Issue 6, June 2017

Corticotropin-releasing hormone and copeptin as acute stress markers in serial cerebrospinal fluid - first evidence for non-response during insulin hypoglycemia test

\section{Background}

In humans, stress response is mediated by the hypothalamic-pituitary-adrenal (HPA) axis and the sympathetic nervous system (1). Their dysfunctions have been hypothesized to be associated with the pathophysiology of stress-related psychiatric diseases such as mood and anxiety disorders (2). Corticotropin-releasing hormone (CRH) and its cofactor arginine vasopressin (AVP), also named antidiuretic hormone (ADH), serve as the most important hypothalamic hormones of the HPA axis to stimulate adrenocorticotropic hormone (ACTH) and consecutively cortisol. Therefore, a valid assessment of both CRH and AVP might directly quantify an individual's current stress level.

Because CRH in plasma mostly stems from intestinal origin, its measurement in cerebrospinal fluid (CSF) as a putative marker of CNS endocrine function has been established and its serial assessment via a lumbar subarachnoidal catheter offers possibilities for dynamic measurement (3). CSF CSH and CSF AVP concentrations (single-point measurements) were reported to predict a major amount of the variation in the ACTH response to exogenously administered CRH in man (4). However, so far there are only few human studies investigating the response of serial CSF $\mathrm{CRH}$ to pharmacological or psychological stimulation $(5,6)$ and CSF AVP has not been measured in this context. Insulin hypoglycemia test offers a powerful stimulus for the hypothalamic release of both secretagogues, as shown in preclinical studies (7). As a reliably assessed and stable surrogate parameter for AVP in plasma, CTproAVP, also termed copeptin, has recently been established (8). Our group detected copeptin also in human CSF (Wiedemann, personal communication). Here, we investigated in a pilot study whether serial CSF concentrations of CRH and copeptin alter during an insulin hypoglycemia test in healthy man and we hypothesized an increase of both.

\section{Methods}

\subsection{Participants}

Five young men (mean age 24,6 years, range $24-26$; mean body mass index 25,7 $\mathrm{kg} / \mathrm{m}^{2}$, range $24,2-27,2$ ) were studied. Neither of them smoked tobacco since it is reported to be associated with lower CSF CRH (5). All participants were healthy according to medical history including epileptic seizures and endocrine dysfunctions, physical examination, standard blood tests and urinary drug screen. They did not take any prescription and nonprescription drugs. Current or prior psychiatric disorders were excluded on the basis of the Structured Clinical Interview for the DSM-IV (SCID), axis I. With the help of the SCID post-traumatic stress disorder modules trauma screen a history of major psychological trauma was tested negative in each subject. None had a history of shift work or transcontinental flights during the past three months. Written informed consent was obtained from all after full oral and written explanation of the purpose and procedures of the investigation. This study had been approved by the Ethical Committee of the Medical Board Hamburg afore.

\subsection{Procedure}

Subjects were studied from 08:00 until 14:00 after over-night fasting. They lay in a supine position in a sound proof private room and were not allowed to sleep, eat or drink. An intravenous cannula was placed into a forearm vein at 08:00 kept open by 50 $\mathrm{ml} / \mathrm{h}$ normal saline. Subsequently, after 
applying local anesthesia, a subarachnoidal catheter (Spinocath $\AA$, B. Braun Melsungen AG, Melsungen, Germany) was inserted through the lumbar interspace $(3-4 ; 4-5)$ by a trained anesthesiologist. An intravenous injection of human insulin (0.1 IE/kilograms body weight) in $10 \mathrm{ml}$ of $0.9 \%$ sodium chloride was injected at 11:02 in a singleblind manner. One physician was attendant at all times. We defined responding to the insulin hypoglycemia test (IHT) as a drop of blood sugar below $40 \mathrm{mg} / \mathrm{dl}$ or below $50 \%$ of the initial value of blood sugar after insulin injection, accompanied by at least sweating as a clinical symptom. From 10:40 to $14: 00$ every 20 minutes $2 \mathrm{ml}$ of CSF were asseted through the subarachnoidal catheter (after discarding $0.5 \mathrm{ml}$ of dead space CSF volume) in order to determine $\mathrm{CRH}$ and copeptin concentrations in CSF. In the same time manner, $10 \mathrm{ml}$ of blood were drawn every 20 minutes for the measurement of blood sugar, ACTH and cortisol (and stored at $-80{ }^{\circ} \mathrm{C}$ ). Both CSF and blood samples were immediately filled into prechilled tubes and stored at $-80^{\circ} \mathrm{C}$ until analysis. Probands were regularly asked for clinical symptoms of hypoglycemia such as sweating, increased appetite, tachycardia, neurologic symptoms like confusion, dizziness and somnolence.

\subsection{Endocrine analyses}

CSF concentrations of $\mathrm{CRH}$ were directly measured with a radioimmunoassay and hCRH was used as a standard and N-tyrhCRH as tracer after labeling with I-125, as previously reported (9). Intra- and interassay coefficients of variation were below $9 \%$, lower limit of detection was $20 \mathrm{pg} / \mathrm{ml}$. Concentrations of copeptin were measured in $50 \mu \mathrm{l}$ of CSF in a sandwich immunoluminometric assay (Brahms CTproAVP, Thermo Scientific, Hennigsdorf, Germany), which uses one polyclonal and one monoclonal antibody. The analytical assay-sensitivity is $0,4 \mathrm{pmol} / \mathrm{L}$, the functional assay-sensitivity is below $1 \mathrm{pmol} / \mathrm{L}$. A single tube luminometer (Lumat $^{\mathrm{R}}$ LB 9508 , Berthold Technologies, Bad Wildbad, Germany) was used. Plasma cortisol concentrations were determined in $25 \mu \mathrm{l}$ serum using a radioimmunoassay with the nucleotid I-125 (DRG, Marburg, Germany) and ACTH by an immunometric assay with the nucleotid I125 (ACTH IRMA, DiaSorin Deutschland $\mathrm{GmbH}$, Dietzenbach, Germany) as reported previously (10).

\section{Results}

\subsection{Side effects and complications}

Out of five subjects, data could be assessed in three. In two cases, no data were obtained due to a complete lack of CSF delivery in one subject and a missing CSF delivery after 11:00 in the other one. No complications (i.e. seizures, unconsciousness) were observed upon insulin injection. Three of five subjects developed mild postural headache within 12 hours of the catheter placement, which fully disappeared the following days. No epidural blood patch was needed for recovery.

\subsection{Hypoglycemic response}

Of the three subjects in which the lumbar catheter delivered CSF throughout, all displayed some typical clinical symptoms of insulin hypoglycemia such as increased appetite, sweating and fatigue within $40 \mathrm{~min}$ after intravenous insulin injection. However, only two subjects fully responded to IHT by showing a significant hypoglycemia in periphery blood tests accompanied by sweating, while the non-responder (subject 3) demonstrated a scarce drop of blood sugar by $44 \%$ after $40 \mathrm{~min}$ and lacked sweating. 
Medical Research Archives, Vol. 5, Issue 6, June 2017

Corticotropin-releasing hormone and copeptin as acute stress markers in serial cerebrospinal fluid - first evidence for non-response during insulin hypoglycemia test

\subsection{Peripheral HPA axis activation}

Plasma ACTH showed an 11-fold increase in subject 1 and a 17-fold increase in subject 2 within 40 minutes after intravenous insulin injection whereas no change was observed in the IHT nonresponder (subject 3). Plasma cortisol concentrations increased accordingly in the responders. Concentrations of plasma ACTH and plasma cortisol are both displayed in Fig. 1.
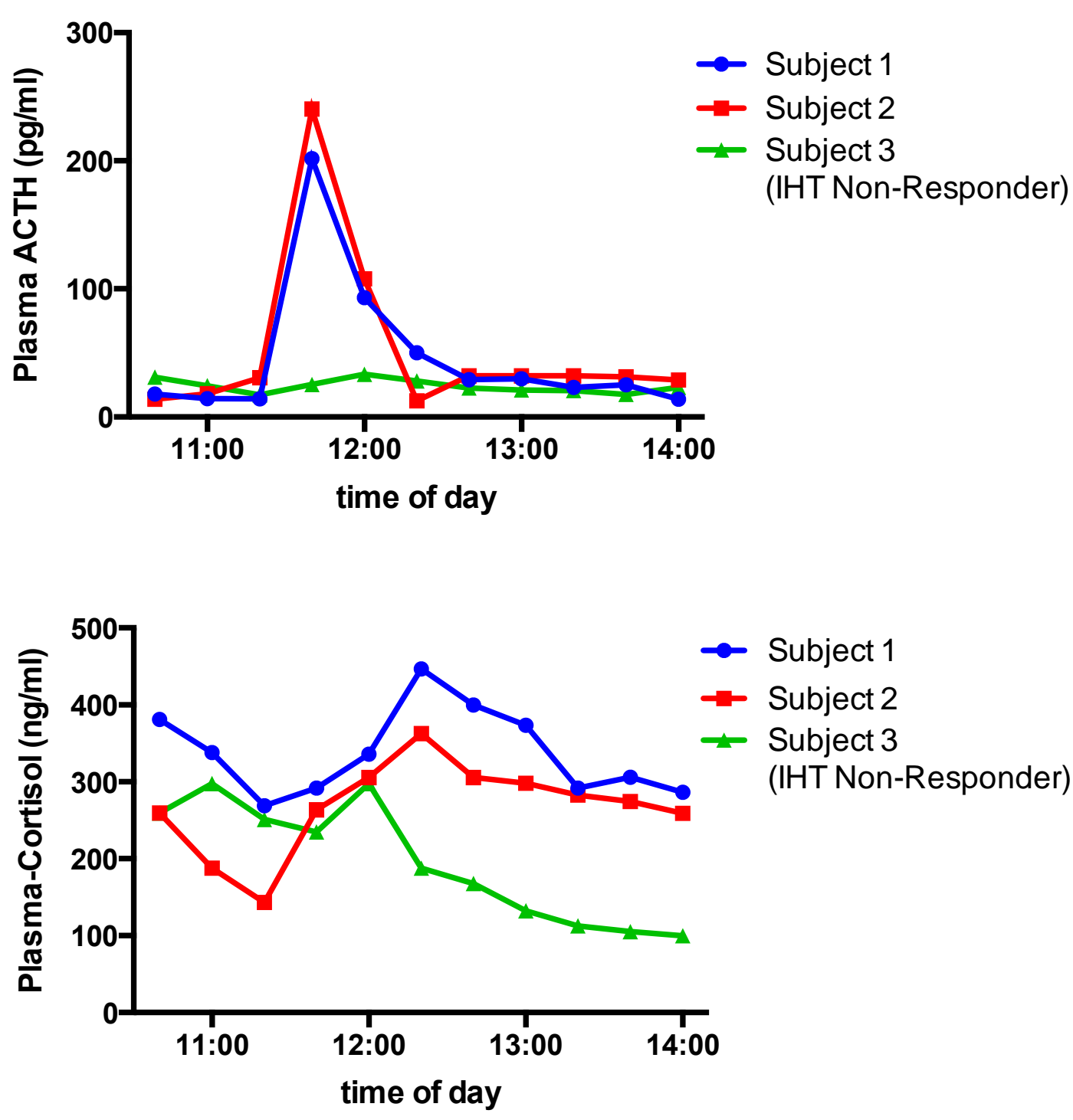

Figure 1. Plasma ACTH and plasma cortisol in three subjects undergoing insulin hypoglycemia (0.1 IE/kilograms body weight of human insulin being injected at 11:02). 
Medical Research Archives, Vol. 5, Issue 6, June 2017

Corticotropin-releasing hormone and copeptin as acute stress markers in serial cerebrospinal fluid - first evidence for non-response during insulin hypoglycemia test

\subsection{CRH and copeptin in serial CSF}

Despite the clear-cut peripheral reaction, neither our hypothesized increase of CSF CRH nor of CSF copeptin was seen in the two responders (Fig. 2). The nonresponder showed a similar time concentration curve. An unexplained isolated secretion pulse of both parameters was observed at 12:40 in subject 2 . A slight trend towards higher concentrations of both substances with the course of time can be observed.
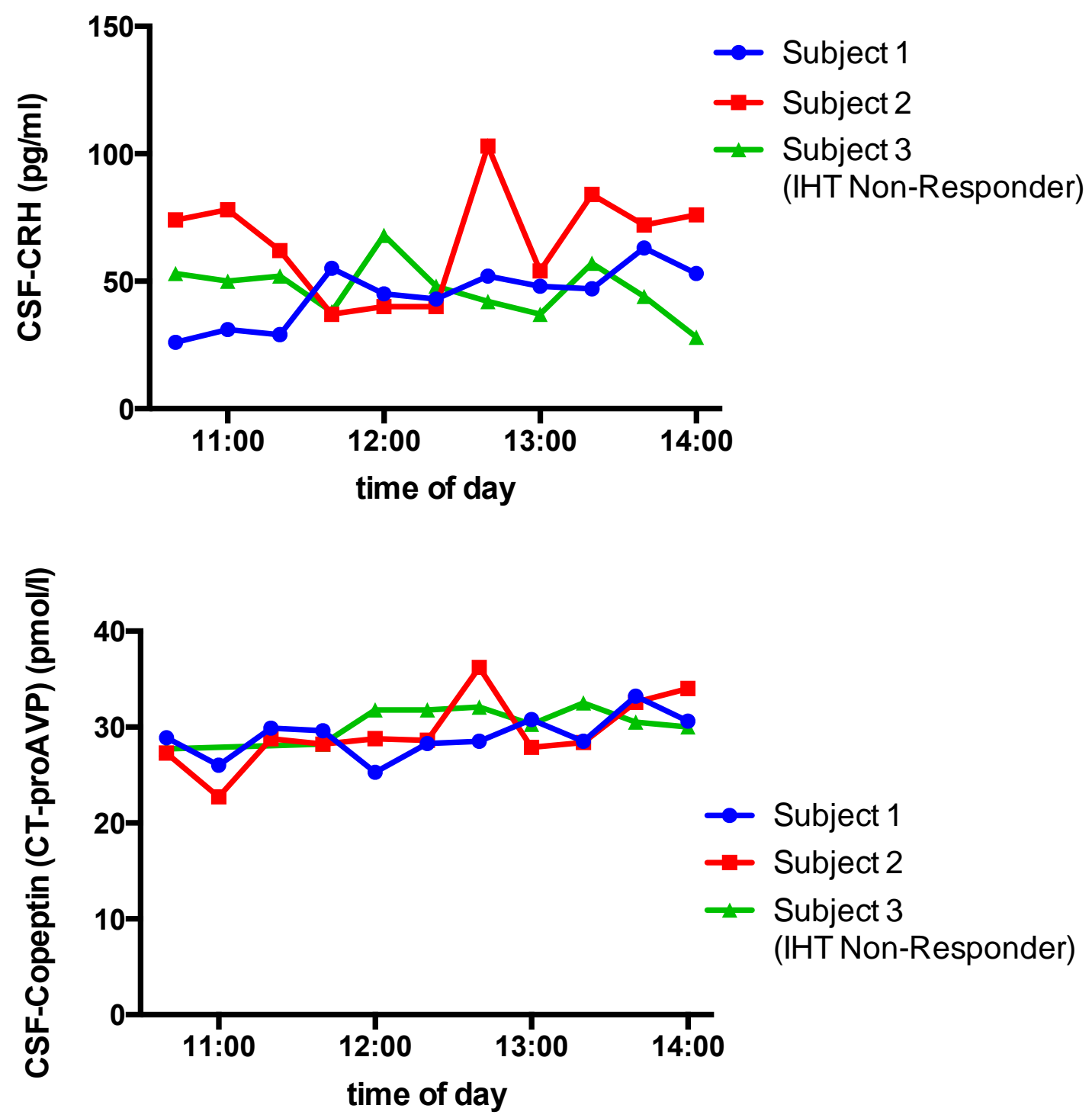

Figure 2. $\mathrm{CRH}$ and copeptin in $\mathrm{CSF}$ in three subjects undergoing insulin hypoglycemia (0.1 IE/kilograms body weight of human insulin being injected at 11:02). 
Medical Research Archives, Vol. 5, Issue 6, June 2017

Corticotropin-releasing hormone and copeptin as acute stress markers in serial cerebrospinal fluid - first evidence for non-response during insulin hypoglycemia test

\section{Discussion}

To our knowledge, this is the first study investigating $\mathrm{CSF} \mathrm{CRH}$ and $\mathrm{CSF}$ copeptin in man during insulin-induced hypoglycemia. Contrary to our hypothesis, we report first evidence that CSF CRH and CSF copeptin do not respond to insulin hypoglycemia in healthy young men despite considerable peripheral HPA axis activation. CSF copeptin has not been measured before.

Recently, Geracioti at al. (11) measured CRH in serial CSF after presenting a stressful psychological suprahypophysial stimulus. Similar to us, they unpredictedly observed a missing increase in serial CSF $\mathrm{CRH}$ in patients with posttraumatic stress disorder during and after exposure to traumatic stimuli. They postulated that the unexpected nonappearance of a rise in CSF $\mathrm{CRH}$ may be explained by stress-induced uptake of CSF $\mathrm{CRH}$ into brain tissue, increased $\mathrm{CRH}$ utilization, increased $\mathrm{CRH}$ degradation or to an acute stress-related inhibition or suppression of CRH secretion mechanisms that could also explain our findings after IHT. Analogously, CSF CRH has been measured after pharmacological suprahypophysial stimuli: Vytilingham and colleagues found a lack of CSF $\mathrm{CRH}$ response in man despite peripheral stimulation of the HPA axis by administration of the potent hypothalamic CRH-releaser naloxone (6). The same pattern was seen in rhesus monkeys, where both metyrapone and physiostigmine did not lead to an increase of CRH in serial CSF despite a HPA axis activation (12).

In addition, we neither had seen acute suppressive effects of cortisol on CSF CRH in a previous study (13). All of these results further support that CSF $\mathrm{CRH}$ does not mirror acute HPA axis activity because it may not reflect neurons projecting from the PVN to the median eminence but rather non-HPA CRH. Altogether the sources and dynamics of CSF CRH still remain unclear, but it has been hypothesized that $\mathrm{CRH}$ neurons in limbic, cortical and brainstem regions altogether contribute to the CSF CRH pool $(14,5)$.

Our novel finding of detectable copeptin in CSF - although not modulated by IHT stimulus in our impressionistic data - encourages further study of its potential regulatory role in health and disease.

\section{Acknowledgement}

We thank Mrs. Iris RemmlingerMarten, Mrs. Sandra Schwentesius and Mrs. Johanna Stalla for their expert technical help. 
Medical Research Archives, Vol. 5, Issue 6, June 2017

Corticotropin-releasing hormone and copeptin as acute stress markers in serial cerebrospinal fluid - first evidence for non-response during insulin hypoglycemia test

\section{References}

1. Chrousos, GP, 1998. Stressors, stress, and neuroendocrine integration of the adaptive response. The 1997 Hans Selye Memorial Lecture. Annals of the New York Academy of Sciences 1998; 851: 311-35.

2. deKloet, CS, Vermetten, E, Geuze, E, Kavelaars, A, Heijnen, CJ, Westenberg, HGM, 2006. Assessment of HPA-axis function in posttraumatic stress disorder: Pharmacological and non-pharmacological challenge tests, a review. Journal of Psychiatric Research 40: 55067.

3. Geracioti, TD, Orth, DN, Ekhator, NN, Blumenkopf, B, Loosen, PT, 1992. Serial cerebrospinal fluid corticotropin-releasing hormone concentrations in healthy and depressed humans. Journal of Clinical Endrocrinology and Metabolism 74: 1325-1330.

4. Newport, DJ, Heim, C, Owens, MJ, Ritchie, JC, Ramsey, CH, Bonsall, R, Miller, AH, Nemeroff, CB, 2003. Cerebrospinal fluid corticotropinreleasing factor (CRF) and vasopressin concentrations predict pituitary response in the CRF stimulation test: a multiple regression analysis. Neuropsychopharmacology 28: 569-76.

5. Geracioti, TD, Loosen, PT, Orth, DN, 1997. Low cerebrospinal fluid corticotropin-releasing hormone concentration in eucortisolemic depression. Biological Psychiatry 42: 166-174.

6. Vytlilingham, M, Anderson, GM, Owens, MJ, Halaszynski, TM, Bremner, JD, Carpenter, LL, Henninger, GR, Nemeroff, DB, Charney, DS, 2000. Cerebrospinal fluid corticotropin-releasing hormone in healthy humans: effects of yohimbine and naloxone. Journal of Clinical Endrocrinology and Metabolism 85: 4138-4145.

7. Caraty A, Grino, M, Locatelli, A, Guillaume, V, Boudouresque, F, ConteDevoix, B, Oliver, C, 1990. Insulininduced Hypoglycemia Stimulates Corticotropin-releasing Factor and Arginine Vasopressin Secretion into Hypophysial Portal Blood of Conscious, Unrestrained Rams. Journal of Clinical Investigation 85: 1716-1721.

8. Morgenthaler, NG, Muller, B, Struck, J, Bergmann, A, Redl, H, et al., 2007. Copeptin, a stable peptide of arginine vasopressin precursor, is elevated in hemorrhagic and septic shock. Shock 28: 219-226.

9. Stalla, GK, Hartwimmer, J, Schopohl J, von Werder K, Müller, AO, 1986. Intravenous application of ovine and human corticotropin releasing factor (CRF): ACTH, cortisol and CRF levels. Neuroendocrinology 42: 1-5.

10. Kellner, M, Muhtz, C, Demiralay, C, Husemann, J, Koelsch, W, Yassouridis, A, Wiedemann, K, 2009. The selective serotonin re-uptake inhibitor escitalopram modulates the panic response to cholecystokinin tetrapeptide in healthy men depending on 5-HTTLPR genotype. Journal of Psychiatric Research 43: 6428.

11. Geracioti, TD, Baker, DG, Kasckow, JW, Strawn, JR, Jeffrey Mulchahey, J, Dashevsky, BA, Horn, PS, Ekhator, NN, 2008. Effects of trauma-related audiovisual stimulation on cerebrospinal fluid norepinephrine and corticotro- 
Medical Research Archives, Vol. 5, Issue 6, June 2017

Corticotropin-releasing hormone and copeptin as acute stress markers in serial cerebrospinal fluid - first evidence for non-response during insulin hypoglycemia test

pinreleasing hormone concentrations in post-traumatic stress disorder. Psychoneuroendocrinology 33: 416- 424.

12. Kalin, NH, Shelton, SE, Barksdale, CM, Brownfield, MS, 1987. A diurnal rhythm in cerebrospinal fluid corticotropinreleasing hormone different from the rhythm of pituitary-adrenal activity. Brain Research 426: 385-391.

13. Kellner, M, Salzwedel, C, Wortmann, V, Urbanowicz, T, Boelmans, K, Yassouri- dis, A, Stalla, GK, Wiedemann, K, 2013. No acute suppression of cerebrospinal fluid corticotropin-releasing hormone in man by cortisol administration. Psychiatry Research 210: 662-664.

14. Garrick, NA, Hill, JL, Szele, FG, Tomai, TP, Gold, PW, Murphy, DL, 1987. Corticotropin-releasing factor: a marked circadian rhythm in primate cerebrospinal fluid peaks in the evening and is inversely related to the cortisol circadian rhythm. Endocrinology 121: 1329-1334. 